\title{
Lewis Base-catalyzed Three-Component Strecker Reaction On Water. An Efficient Manifold for the Direct $\alpha$-Cyanoamination of Ketones and Aldehydes
}

\author{
Fabio Cruz-Acosta, ${ }^{a, b}$ Alicia Santos-Expósito, ${ }^{a}$ Pedro de Armas, ${ }^{* a, b}$ and Fernando García-Tellado, $* a, b$ \\ ${ }_{5}$ Received (in $\left.X X X, X X X\right)$ Xth $X X X X X X X X X 200 X$, Accepted Xth $X X X X X X X X X 200 X$ \\ First published on the web Xth $X X X X X X X X X 200 X$ \\ DOI: 10.1039/b000000x
}

The first three-component organocatalyzed Strecker reaction operating on water has been developed. The manifold utilizes ${ }_{10}$ ketones (aldehydes) as the starting carbonyl component, aniline as the primary amine, acetyl cyanide as the cyanide source and N,N-dimethyl cyclohexylamine as the catalyst.

The three-component reaction of an aldehyde or ketone, a primary amine and a cyanide ion, (Strecker reaction, S-3CR), ${ }^{1}$ 15 constitutes one of the most efficient manifolds for the synthesis of $\alpha$-amino nitriles, versatile precursors of $\alpha$-amino acids. ${ }^{2}$ Since its discovery in 1850 , a large survey of modified reaction conditions and different cyanating agents has been described. ${ }^{3}$ In spite of these advances, the development of simple, efficient and general 20 multicomponent manifolds utilizing ketones remains a challenge. ${ }^{4}$ During the last two years, some interesting advances have been reported including solvent-free systems, ${ }^{5}$ gallium (III) triflate catalyzed reactions, ${ }^{6}$ the use of fluorous solvents, ${ }^{7}$ solid acid catalysts ${ }^{8}$ and high pressures. ${ }^{9}$ However, all of them utilize 25 toxic and expensive trimethyl silyl cyanide (TMSCN) as the cyanide source and only three of them display a wide ketone scope (iron, ${ }^{5 \mathrm{~b}}$ gallium ${ }^{6}$ and solid acid $^{8}$ catalyzed reactions). Acyl cyanides ${ }^{10}$ have emerged as less toxic and efficient alternatives to TMSCN (eqn (1)). ${ }^{11}$ Therefore, its use in catalytic S-3CR 30 involving ketones remains an unsolved challenge. ${ }^{11 a}$ Among other possibilities, the development of water-compatible S-3CR manifolds involving this reagent and ketones would be advantageous.

35

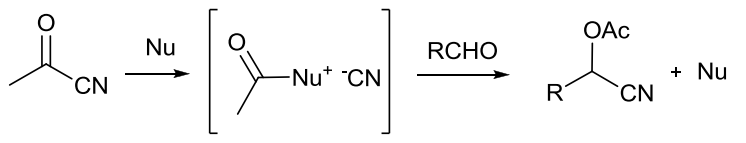

A large list of examples in recent chemical literature shows that organic reactions performed at the interface organic-water 40 (on water) $^{12}$ are not only typically faster but also display novel reactivity profiles and selectivity. ${ }^{13}$ A theoretical model based on the unique structure of the water-organic interface has been recently postulated to explain this effect. ${ }^{14}$ The model relates these effects with the special structure of water at the oil-water 45 interface, which maintains an approximately $25 \%$ of water molecules in the form of tri-coordinated water, with their free $\mathrm{OH}$ group protruding into the organic phase. ${ }^{14}$ The unmet hydrogen bonding demand of these tri-coordinated water molecules on the surface of the water phase provides an immediate energy
50 advantage to the reactants capable of generating hydrogen bonding for catalysis. We hypothesized that the S-3CR of liphophilic ketones with primary amines and acetyl cyanide could be one of such catalyzed reactions on water because: 1) ketimines (intermediates) are expected to form stronger and better $\mathrm{H}$-bonds 55 than their parent ketones (reactants) (selective ketimine activation); 2) ketimine formation is catalyzed by H-bond interactions (carbonyl activation); 3) cyanide generation involving Lewis base catalysis is expected to be $\mathrm{H}$-bond favored (increased carbonyl reactivity). As a proof of concept, we report ${ }_{60}$ herein the first Lewis base-catalyzed S-3CR of ketones operating on water. The manifold utilizes a tertiary amine as the catalyst, acetyl cyanide as the cyanide source and aniline as the primary amine. To the best of our knowledge, no precedents for this basecatalyzed MCR have been reported to date.

65 We accordingly begun our investigation studying the multicomponent reaction of 3-octanone (2a) with aniline and acetyl cyanide in the presence of catalytic amounts of liphophilic $\mathrm{N}, \mathrm{N}$-dimethyl cyclohexylamine, (a common structural component of many Lewis base-based organocatalysts), under on water 70 conditions (eqn (2)). Because hydrophobic effects are known to play an important role in the reactions performed at the water surface, we directly used brine instead of water as the reaction medium. Thus, the vigorous stirring of a salt-saturated aqueous suspension of stoichiometric mixtures of ketone, aniline and

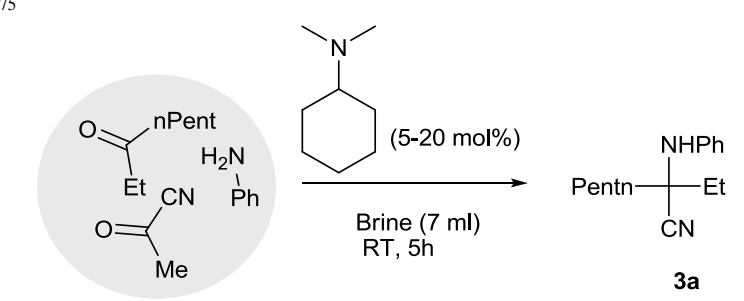

acetyl cyanide and $20 \mathrm{~mol} \%$ of the tertiary amine, afforded, after $5 \mathrm{~h}$ at room temperature, the corresponding $\alpha$-aminonitrile 80 derivative $3 \mathbf{a}$ in $49 \%$ yield. Because acetyl cyanide is known to afford base-catalyzed homo-O-acylation with release of one unit of cyanide anion, ${ }^{10 \mathrm{~b}}$ we increased the amount of this reactant to two equivalents. Under these new conditions, the multicomponent reaction turned almost quantitative $(\geq 95 \%$ 85 yield). Remarkably, the reaction did not produce $\mathrm{N}$-acylated $\alpha$ aminonitriles which indeed constitute the main products in organic media using this cyanide source. ${ }^{11}$ Other tertiary amines spanning different pKa's and lipophobicities (N,N-diisopropil 
ethylamine, DABCO, pyridine and quinine) were also good catalysts for this reaction (see ESI for details). Further experimentation showed that the catalyst charge could be reduced to a more practical $5 \mathrm{~mol} \%$ without yield erosion. Finally, the 5 efficiency of the manifold was considerably reduced in the absence of catalyst, affording product 3a in less than $10 \%$ yield.

Once the experimental conditions were standardized, we next studied the scope of this S-3CR with regard to the ketone (Table 1). Both aromatic and aliphatic cyclic or acyclic ketones were 10 good substrates for this reaction. Acyclic ketones were conveniently transformed into the $\alpha$-aminonitrile derivatives with independence of the chain length (entries 1-7). Branched aliphatic ketone $\mathbf{2 d}$ afforded the corresponding $\alpha$-aminonitrile $\mathbf{3 d}$ in good yield (entry 5). Ketone $\mathbf{2 h}$ featuring a cyclohexane ring and a 15 phenyl ring showed a diminished reactivity affording compound 3h in low yield and under a prolonged reaction time (entry 9). Even acetophenone (2g), a scarcely reactive ketone, gave the corresponding cyanoamination product $\mathbf{3 g}$ in good yield (entry 8). Most reactive cyclic ketones proved to be excellent substrates for 20 this manifold (entries 10-11). Remarkably, products coming from the C-acylation of the enamine tautomer of the ketimine intermediate were not detected under these standardized conditions. Finally, the manifold could be performed at a 25 mmol scale without a severe erosion of yield (entry 2). ${ }_{25}$ Encouraged by these results we extended our findings to the S3CR with aldehydes. Although a large array of methodologies are available for this reaction, ${ }^{3}$ water-compatible multicomponent manifolds remain significantly less explored. ${ }^{15}$ Under the same experimental conditions, a wide set of aldehydes could be 30 transformed into the corresponding $\alpha$-aminonitrile derivatives by the three-component manifold (Table 1, entries 12-21). Both aliphatic and aromatic aldehydes $\mathbf{2 k - t}$ were efficiently transformed into the corresponding $\alpha$-aminonitriles $3 \mathbf{k}-\mathbf{t}$ in a high overall average yield. Even acetaldehyde (2k), a hydrophilic 35 aldehyde, could be transformed into the corresponding aminonitrile $\mathbf{3 k}$ in a modest-good yield (entry 12). A chain effect was observed with aldehyde $\mathbf{2} \mathbf{p}$ featuring a linear $\mathrm{C}_{10}$ carbon alkyl chain. After $1 \mathrm{~h}$ of reaction, only $30 \%$ of the corresponding cyanoamination compound $\mathbf{3 p}$ could be obtained (entry 17). This 40 chain length seems to be large enough to induce hydrophobic associations affording reduced aldehyde reactivity. On the other hand, heptanal (2o), featuring a linear $\mathrm{C}_{6}$ carbon alkyl chain, generated compound 30 in very good yield (entry 16). Aromatic aldehydes 2s-t were fully transformed into the corresponding 45 products 3s-t practically in quantitative yield (entries 20-21). Remarkably, we could not detect the formation of cyanohydrins 4 coming from a competitive addition of cyanide on the starting aldehydes (see ESI for details). This observed chemoselectivity reinforces our previous hypothesis that the imines would form 50 stronger and better H-bonds than aldehydes or ketones with the $\mathrm{H}$-bond network of the tri-coordinated structural water molecules at the interface, and therefore, they would be more reactive than their parent carbonyl compounds (selective imine activation).

The effect of the hydrophobicity associated with the primary 55 amine was studied using the reation of acetophenone $(\mathbf{2 g})$ and aniline, 4-n-butoxyaniline and 4-n-heptylaniline as the amine sources. Under the estandarized conditions, the more liphophilic 4-n-butoxy and 4-n-heptylanilines showed a higher efficiency than aniline, affording the corresponding $\alpha$-cyanoamination 60 compounds in practically quantitative yields $(71 \%$ yield using aniline).

Table 1. N,N-dimethyl cyclohexylamine catalyzed S-3CR on brine.

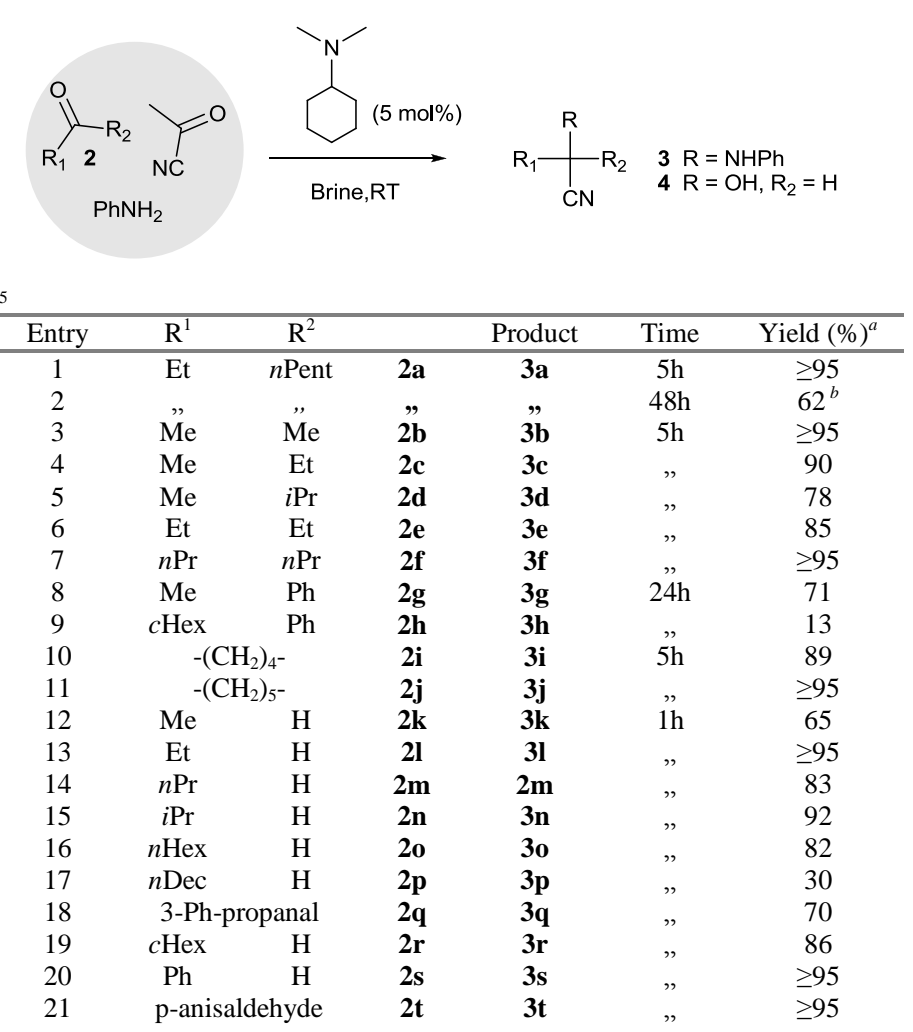

${ }^{a}$ Yields of analytically pure products. ${ }^{b}$ Ketone $(25 \mathrm{mmol})$, aniline (25 mmol), acetyl cyanide $(50 \mathrm{mmol}), \mathrm{N}, \mathrm{N}$-dimethyl cyclohexylamine (5 mol\%), brine $(250 \mathrm{ml}), \mathrm{RT}$. Non optimized yield

70 The basic character of this catalyzed S-3CR was confirmed by the following experimental facts:

1) Manifold requieres two equivalents of acyl cyanide to be effective, which is coherent with a cyanide anion release launched by a Lewis base-catalyzed dimerization process, ${ }^{10}$ 75 and it rules out a direct cyanide anion release by nucleophilic attack of the Lewis base on the acyl center of the reagent (eqn (1)) or through an alternative base-catalyzed ketene formation. 2) The highly basic and non-nucleophilic N,N-diisopropyl ethylamine catalyzes the reaction of $\mathbf{2 a}$ with aniline, generating 80 the cyanoamination compound $\mathbf{3 a}$ in $85 \%$ yield (Table 2 , entry 1 ). 3) Substitution of acetyl cyanide by the less $\alpha-\mathrm{CH}$ acidic butyryl cyanide raises the pKa's threshold for the catalyst: while pyridine roughly catalyzes the reaction $(12 \%)$, the more basic $\mathrm{N}, \mathrm{N}$ dimethyl cyclohexylamine affords product 3a with moderate-to85 good efficiency (69\%) (Table 2, entries 2 and 3). Observe that in the absence of catalyst, the manifold still generates a background amount of $\alpha$-amino nitrile $\mathbf{3 a}(\leq 10 \%)$ (entry 4$)$. If we compare the yields in the absence of catalyst (acetyl cyanide) and that obtained with pyridine (butyryl cyanide), both are in the same 90 range; that is, they represent the background yield for the noncatalyzed S-3CR. Finally, benzoyl cyanide, lacking $\alpha$-protons, 
drastically reduces the efficiency of the manifold to the background level $(\leq 10 \%)$ (entry 5$)$.

Table 2. Basic character of the S-3CR manifold.

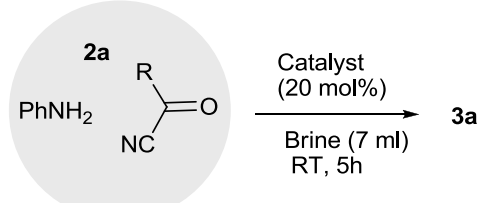

\begin{tabular}{cccc}
5 & & & \\
\hline Entry & $\mathrm{R}$ & Catalyst & ${\text { Yield }(\%)^{a}}^{a}$ \\
\hline 1 & $\mathrm{Me}$ & N,N-diisopropyl ethylamine & 85 \\
2 & $n \mathrm{Pr}$ & N,N-dimethyl cyclohexylamine & 69 \\
3 & $n \mathrm{Pr}$ & Pyridine & 12 \\
4 & $\mathrm{Me}$ & None & $\leq 10$ \\
5 & $\mathrm{Ph}$ & $\mathrm{N}, \mathrm{N}-$ dimethyl cyclohexylamine & $\leq 10$ \\
& \\
&
\end{tabular}

A mechanistic proposal accounting for the experimental results is outlined in Scheme 1. The ketone (aldehyde) reacts with 10 the primary amine to give the corresponding ketimine (aldimine). To be chemically efficient, this reaction requires a dehydrating agent to shift the equilibrium toward the imine product. Herein, the own manifold realizes this task removing the water generated in the condensation from the organic phase (drop) to the bulk 15 water, shifting the ketone-imine equilibrium toward the imine side. This counterintuitive physicochemical behaviour is very remarkable and it offers a powerful manner to perform "dry" organic chemistry in water. Next, the imine reacts with the cyanide anion generated in the Lewis base-catalyzed dimerization 20 of acetyl cyanide to give the corresponding amide intermediate, which in turn is neutralized by the Lewis base conjugated acid to give the $\alpha$-amino nitrile derivative 3 with regeneration of the Lewis base to reinitiate the cycle.

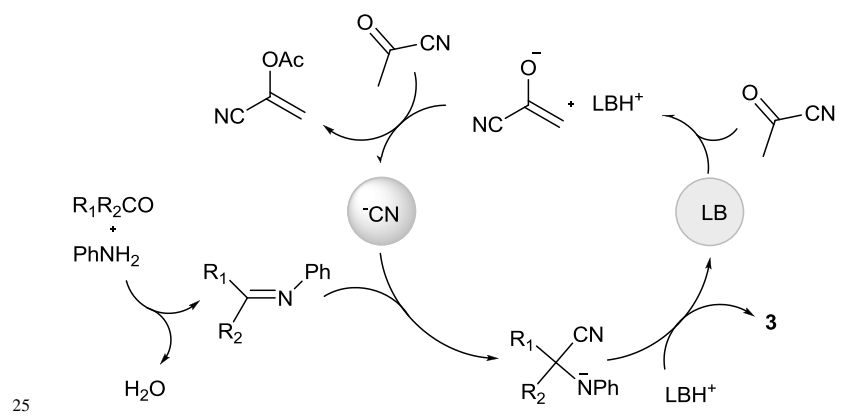

Scheme 1. A mechanistic proposal. $(\mathrm{LB}=$ Lewis base $)$

Acknowledgements. This work was supported by the Spanish by the Spanish Ministerio de Ciencia e Innovación 30 and the European Regional Development Fund (CTQ200509074-C02-02 and CTQ2008-06806-C02-02), the Spanish MSC ISCIII (RETICS RD06/0020/1046) and Fundación Instituto Canario de Investigación del Cáncer (FICICREDESFAC). F.C.-A thanks CSIC for a predoctoral JAE 35 grant.

\section{Notes and references}

${ }^{a}$ Department of Química Biológica y Biotecnología

Instituto de Productos Naturales y Agrobiología

Consejo Superior de Investigaciones Científicas

40 Astrofísico Francisco Sánchez 3

38206 La Laguna-Tenerife-Spain

Fax: (+)34922-260135

E-mail:fgarcia@ipna.csic.es: parmas@ipna.csic.es

Homepage: http://www.ipna.csic.es/departamentos/qbb/qb/

${ }_{45}{ }^{b}$ Instituto Canario de Investigación del Cáncer, Spain, www.icic.es

$\dagger$ Electronic Supplementary Information (ESI) available: experimental section and physical data for compounds 3h. See DOI: $10.1039 / \mathrm{b} 000000 \mathrm{x} /$

$50 \doteqdot$ General procedure. To a stirred $(250 \mathrm{rpm})$ salt-saturated aqueous solution (brine) $(7 \mathrm{ml})$ is sequentially added acetyl cyanide $(1.4 \mathrm{mmol})$, $\mathrm{N}, \mathrm{N}$-dimethyl cylclohexylamine $(0.035 \mathrm{mmol})$, carbonyl compound 2a $(0.70 \mathrm{mmol})$ and aniline $(0.70 \mathrm{mmol})$ (dropwise) to form a biphasic system. The stirring rate is increased to $1200 \mathrm{rpm}$ and after a few minutes, 55 the biphasic system breaks into small drops. After $5 \mathrm{~h}$ ( $1 \mathrm{~h}$ for aldehydes) the reaction is quenched by addition of dichloromethane and the organic materials are recovered in dichloromethane. Concentration and flash chromatography (ethyl acetate-hexanes: 20/80) yields pure compound 3a: oil, ${ }^{1} \mathrm{H}$ NMR $\left(400 \mathrm{MHz}, \mathrm{CDCl}_{3}\right): \delta=0.91\left(\mathrm{t},{ }^{3} J(\mathrm{H}, \mathrm{H})=7 \mathrm{~Hz}, 3 \mathrm{H}\right), 1.07(\mathrm{t}$,

$\left.60{ }^{3} \mathrm{~J}(\mathrm{H}, \mathrm{H})=7.5 \mathrm{~Hz}, 3 \mathrm{H}\right), 1.29-1.39(\mathrm{~m}, 4 \mathrm{H}), 1.45-1.58(\mathrm{~m}, 2 \mathrm{H}), 1.82-2.08$ (m, 2H), 3.5 (bs, NH), 6.86-6.95 (m, 3H), 7.21-7.28 (m, 2H); ${ }^{13} \mathrm{C}$ NMR $\left(100 \mathrm{MHz}, \mathrm{CDCl}_{3}\right) \delta=8.0,13.9,22.4,23.6,30,31.6,36.6,57.3,117.1$, 120.3, 121.1, 129.3 (x3C), 143.8; IR $\left(\mathrm{CHCl}_{3}, \mathrm{~cm}^{-1}\right): 3431(\mathrm{NH}), 2230,5$ (CN); Anal. Calcd. for $\mathrm{C}_{15} \mathrm{H}_{22} \mathrm{~N}_{2}$ : C, 78.21; H, 9.63; N, 12.16. Found: C, 65 78.02; H, 9.79; N, 12.06

1 S. Strecker, Ann. Chem. Parm. 1850, 75, 27.

2 For recent general reviews on the synthesis of $\alpha$-amino acids: a) C. Nájera, J. M. Sansano Chem. Rev. 2007, 107, 4584. b) A. Perdih, M. S. Dolenc, Curr. Org. Chem. 2007, 11, 801.

703 M. Shibasaki, M. Kanai, T. Mita, The catalytic Asymmetric Strecker Reaction in Organic Reactions Vol. 70 (Ed: L. Overman), Hoboken, NJ, 2008, pp. 1-119.

4 a) J. S. Connon, Angew. Chem. Int.. Ed. 2008, 47, 1176 and references cited therein. b) C. Spino, Angew. Chem. Int. Ed. 2004, 43, 1764.

5 a) A. Baeza, C. Najera, J. M. Sansano, Synthesis 2007, 1230. b) For an iron-catalyzed solvent-free process see: N. H. Khan, S. Agrawal, R. I. Kureshy, S. H. R. Abdi, S. Singh, E. Suresh, R. V. Jasra, Tetrahedron Lett. 2008, 49, 640.

${ }_{80} 6$ G. K. S. Prakash, T. Mathew, C. Panja, S. Alconcel, H. Vaghoo, C. Do, G. A. Olah, Proc. Natl. Acad. Sci. U. S. A. 2007, 104, 3703. b) G. K. S. Prakash, C. Panja, C. Do, T. Mathew, G. A. Olah, Synlett 2007, 2395.

7 A. Heydari, S. Khaksar, M. Tajbakhsh, Tetrahedron Lett. 2009, 50, $85 \quad 77$

8 G. K. S. Prakash, T. E. Thomas, I. Bychinskaya, A. G. Prakash, C. Panja, H. Vaghoo, G. A. Olah, Green Chem. 2008, 10, 1105.

9 K. Matsumoto, J. C. Kim, H. Iida, H. Hamana, K. Kumamoto, H. Kotsuki, G. Jenner, Helv. Chim. Acta 2005, 88, 1734.

9010 a) S. Lundgren, E. Wingstrand, C. Moberg, Adv. Synth. Catal. 2007, 349, 364 and references cited therein. b) S. Hüning, R. Schaller, Angew. Chem. Int. Ed. 1982, 21, 36.

11 a) S. Chandra Pan, B. List, Chem. Asian J. 2008, 3, 430 and references cited therein. b) W. Zhang, M. Shi, Org. Biomol. Chem. 2006, 4, 1671. c) S. Lundgren, E. Wingstrand, M. Penhoat, C. Moberg, J. Am. Chem. Soc. 2005, 127, 11592. d) Y. Ishino, M. Mihara, T. Takeuchi, M. Takemoto, Tetrahedron Lett. 2004, 45, 3503. e) T. Watahiki, S. Ohba, T. Oriyama, Org. Lett. 2003, 5, 2679. f) M. Scholl, C.-K. Lim, G. C. Fu, J. Org. Chem. 1995, 60, 6229.

10012 On water refers to the reactions performed with sparingly soluble or insoluble reactants in water. S. Narayan, J. Muldoon, M. G. Finn, V. V. Fokin, H. C. Kolb, K. B. Sharpless, Angew. Chem. Int. Ed. 2005, 44, 3275 .

13 A. Chanda, V. V. Fokin, Chem. Rev. 2009, 109, 725

10514 F. G. Moore, G. L. Richmond, Acc. Chem. Res. 2008, 41, 739. 
15 a) Z.-L. Shen, S.-J. Ji, T.-P. Loh, Tetrahedron 2008, 64, 8159. b) S.

Kobayashi, T. Busujima, Chem. Commun. 1998, 981. 